\title{
LOS TRES PLANOS DE LA TEOLOGÍA
}

\section{THE THREE LEVELS OF THEOLOGY}

Juan Alberto Osorio Torres*

\begin{abstract}
RESUMEN
El texto destaca el rol de la teología que resulta desempeñando el funcionamiento pastoral de una Iglesia particular o diócesis en la comprensión de la necesidad y utilidad del quehacer teológico. No sólo reitera en la función eclesial de la teología, sino que además, advierte sobre todo en la riqueza testimonial de pastores y sobre todo de fieles, cuya pública y profunda comunión da cuenta del hecho de una rotunda opción de los miembros de tal comunidad diocesana: haber decidido subordinar el peso de la estructura y de la tradición, a la urgencia del Evangelio y el proceso emprendido a favor del Reino, a pesar del conflicto y la crisis que ello implica.

Después del Concilio Vaticano II y Medellín, la comprensión y razón de ser de la teología, no solo cuenta con el plano bíblico y patrístico, ni solo con el plano kerygmático, sino además con el plano diocesano, su mejor y más atractiva síntesis históricamente vivenciada.
\end{abstract}

\section{PALABRAS CLAVE:}

Teología, Diócesis, estructura, proceso, pastoral

\begin{abstract}
The text highlights the theology's role that results from performing the pastoral functioning of a Church or diocese in understanding the need and usefulness of its function.

It reiterates not only in the ecclesial function of the theology, but also warms, above all, the testimonial wealth of pastors and the faithful, whose public and profound communion realizes a strong option made by the members of such diocesan community: having decided to subordinate the weight of the structure and tradition to the urgency of the Gospel and the process undertaken for the Kingdom, despite the conflict and the crisis that it implies.

After II Vatican Council and Medellin, the understanding and rationale of the theology not only has the biblical, patristic and kerygmatic level, but also the diocesan level, its best and most attractive historical synthesis experienced.
\end{abstract}

\section{KEYWORDS:}

Theology, Diocese, structure, process, pastoral

Docente ordinario de la Universidad Femenina del Sagrado Corazón: aosorio@unife.edu.pe 
"Un reclamo marcadamente juvenil suele resonar en algunos ambientes parroquiales y estudiantiles, a poco menos de llegar al año 2000: "A la Iglesia le falta renovarse... a la Iglesia le cuesta renovarse...". Esta percepción puede reflejar más que una falla objetiva de la Iglesia el desconocimiento de la experiencia vivida por ésta en los últimos 40 años. El Concilio Vaticano II y las Conferencias Episcopales Latinoamericanas de Medellín, Puebla y Santo Domingo respondieron entonces a una similar preocupación, y no pueden dejar de ser hoy una referencia clave de la renovación en aquellas décadas." ${ }^{1}$

Ha sido por éste enfoque, asumido en la Escuela de Graduados en Ciencias Sociales de la Pontificia Universidad Católica del Perú, con motivo de mi tesis de Maestría (1998), y gracias al estímulo de Monseñor José Dammert y a la asesoría de Manuel Marzal, que pude tomar dos decisiones que sigo manteniendo a partir de entonces: considerar el tema de la diócesis para la tesis doctoral, y responder a las inquietudes y perplejidades de no pocas de mis alumnas de Teología y de Iglesia y Doctrina Social, respecto de la fe, de Dios y de la Iglesia.

En este artículo, que tengo en cuenta ambos datos, voy a hablar del papel que puede jugar el testimonio de los miembros de una diócesis en la comprensión de la teología y de los principios de la Doctrina Social de la Iglesia. Lo haré, a partir de una investigación ya concluida, en la que desde la antropología de la religión, doy cuenta del significado del nacimiento de una diócesis nueva, -la Diócesis de Lurín-, a partir de la experiencia ocurrida en la Arquidiócesis de Lima, en el periodo de 1954 a 1996.

Por esto, me pregunto ¿cómo hacer para que las alumnas no sólo despierten su interés por los temas, ni solo descubran la importancia de los mismos, sino que además, en lo posible, nuestra reflexión filosófica y teológica resulte ofreciéndoles en el curso, un nexo y hasta una fuente de motivaciones que resulte consolidando y enriqueciendo la vocación profesional de sus respectivas carreras? ¿Cómo orientar y fortalecer "el sentido religioso y trascendente -de las alumnas- mediante la reflexión teológica sobre el Dios que nos viene al encuentro y la respuesta libre y comprometida como creyentes"? ${ }^{2}$

¿De qué manera, más próxima y vivencial, la joven, inteligente e independiente, llega a "conocer a Jesucristo a través de sus palabras y obras, su muerte que nos reconcilia con Dios y el valor salvífico de su resurrección"? ¿Con qué enfoque y prácticas contamos los teólogos y filósofos para acompañar a la universitaria a "reconocer a la Iglesia como pueblo peregrino en la tierra, que camina en la esperanza y nos invita a participar en el Reino de Dios, inaugurado por Cristo y que apunta a su plenitud escatológica."?3. ¿Cómo llegamos a coincidir juntos, alumnas y maestros, en

1 Con este primer párrafo introducía mi tesis de maestría en octubre 1998, con la que, desde la antropología de la religión, cumplí con el encargo de José Dammert Bellido, obispo de Cajamarca, quien me envió a la Pontificia Universidad Católica del Perú, con el fin de que pudiera comprender y valorar la cultura campesina y terminara informando con mi tesis de maestría acerca de los "Treinta años de cambios pastorales en la parroquia minera de San Fernando de Hualgayoc y la parroquia rondera de San Carlos de Bambamarca 1963 1993", en las que me hallaba laborando junto con las Religiosas del Sagrado Corazón y el Padre Rolando Estela, a partir de 1981.

2 Objetivo general del Sílabo de Teología I, año 2012

3 Objetivo general del Sílabo de Teología II, año 2012 
que el "sentido ético-social-cristiano", o en que "el fundamento de la Doctrina Social de la Iglesia", es algo más que retórica?4 .

Convencido de que el testimonio de la fe vivida respecto del dogma y de la doctrina teológica, guarda directa proporción con la credibilidad que posee la imagen respecto de las palabras, una de las primeras maneras en que me encuentro intentando resolver la inquietud arriba planteada, es relacionando en paralelo los temas teológicos con la experiencia kerygmática y diocesana actual, reciente o remota de obispos, sacerdotes, laicas y laicos de parroquias de dentro o fuera del país. Como figura en el cuadro siguiente, lo que hago es agregar al básico plano bíblico y patrístico de los temas, el doble explícito plano kerygmático y diocesano. Explico brevemente en qué consisten los dos primeros y me explayo un tanto más en el último.

\section{Cuadro $\mathrm{N}^{\circ} 1$}

\section{PLANOS DE LA TEOLOGÍA}

\begin{tabular}{|c|c|c|c|}
\hline Curso & $\begin{array}{c}\text { Plano } \\
\text { Bíblico-Patrístico }\end{array}$ & Plano Kerygmático & Plano Diocesano \\
\hline Teología I & $\begin{array}{ll}\text { - } & \text { El Hecho Religioso } \\
\text { - } & \text { Revelación y Fe } \\
\text { - } & \text { Pentateuco } \\
\text { - } & \text { Históricos } \\
\text { - } & \text { Proféticos } \\
\text { - Sapienciales }\end{array}$ & & \\
\hline Teología II & $\begin{array}{l}\text { - Encarnación } \\
\text { - Reino de Dios } \\
\text { - Misterio Pascual } \\
\text { - Ascensión } \\
\text { - Pentecostés } \\
\text { - Llamados y enviados } \\
\text { - Parusía }\end{array}$ & & $\begin{array}{l}\text { Comunidad cristiana } \\
\text { - Solidaria } \\
\text { - Crítica } \\
\text { - Pública } \\
\text { Parámetros de } \\
\text { funcionamiento }\end{array}$ \\
\hline $\begin{array}{l}\text { Iglesia y Doctrina } \\
\text { Social }\end{array}$ & 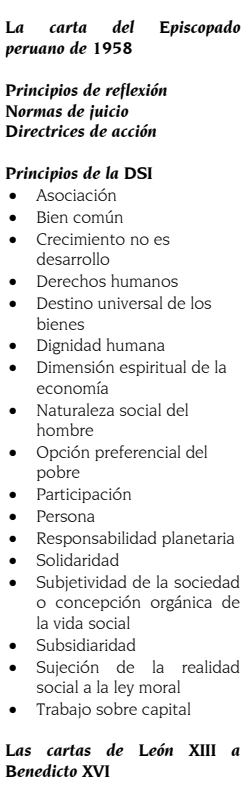 & $\begin{array}{l}\text { Dimensiones: } \\
\text { - Pneumatológica } \\
\text { - Histórico, primordial, } \\
\text { pascual } \\
\text { - Crítica, profética, } \\
\text { pública } \\
\text { - Comunitaria, } \\
\text { solidaria, organizada } \\
\text { - Plural e intercultural } \\
\text { - Cristológica, laical y } \\
\text { femenina }\end{array}$ & $\begin{array}{l}\text { - de comunión entre } \\
\text { pastores y fieles } \\
\text { - al servicio de los más } \\
\text { pobres e indefensos de la } \\
\text { diócesis } \\
\text { Funciones de la Diócesis } \\
\text { - Función jurídica y } \\
\text { organizativa } \\
\text { - Al servicio de la función } \\
\text { pastoral } \\
\text { - Y las tres al servicio de la } \\
\text { función cósmica } \\
\text { Entre estructura y proceso } \\
\text { - No son dilema } \\
\text { - La estructura no existe de } \\
\text { manera independiente } \\
\text { - La estructura al servicio del } \\
\text { proceso }\end{array}$ \\
\hline
\end{tabular}

4 Objetivo general del Sílabo de Iglesia y Doctrina Social, año 2012. 


\section{Plano Bíblico-Patrístico}

El plano bíblico tiene como propósito, revelar la presencia de Dios creador, liberador en la historia de una multitud que tras la búsqueda de la tierra prometida, empieza a construir su identidad y su misión de Pueblo Sacerdotal, Pueblo de Dios. Pueblo de patriarcas y sus esposas, de jueces y reyes, profetas y sabios, a la búsqueda de la fidelidad a la nueva Alianza.

En el primer ciclo de Teología, una vez desarrollado el tema introductorio del Hecho Religioso, el resto del curso se centra en la revelación y la fe experimentada por la vida e historia del pueblo de Israel. Se trata de la historia de Salvación, registrada a lo largo del Antiguo Testamento y esquematizada en siete grandes etapas: aparte de la Pre-existencia, estas siete etapas que contienen los paradigmáticos hechos y personajes son: orígenes, esclavos y fugitivos, errantes, instalados, desterrados, reconstructores y perseguidos. La etapa que denomino como 'instalados', en la que el pueblo vive a partir de que las doce tribus se asientan en Canaán, es la más larga.

Por eso, se subdivide en otras etapas que están marcadas, primero, por el liderazgo de Jueces y luego de los reyes. Esta etapa gloriosa, llega a su fracaso con el cisma y división del único reino en reino del Norte (Israel-Samaria) y el reino del Sur (Judea-Jerusalén) y las posteriores invasiones del reino del Norte por parte de Asiria, y el del Sur, por Babilonia. Estas invasiones traen consigo el destierro del pueblo, que lo va a poner al borde de la extinción. Y que precisamente, a partir de la experiencia de la etapa de desterrados hasta la de los perseguidos, demarca un segundo gran bloque de esta historia, probablemente teológica e históricamente tan y más rica que el primero, y el cual llega a su consumación con el nacimiento de Jesús.

Para el segundo ciclo de teología, los temas giran en torno al nacimiento de Jesús y el origen de la comunidad de apóstoles y discípulos del Resucitado Vencedor de la muerte. Es decir, el desarrollo pasa por la sucesión de siete grandes acontecimiento o misterios, cuyo esquema facilita y sintetiza el conjunto de temas fundamentales a partir de Encarnación, Reino de Dios, Misterio Pascual, Ascensión, Pentecostés, Llamados y enviados y Parusía.

\section{Plano Kerygmático}

El plano kerygmático, tiene como propósito, apreciar y emular, difundir y extender la valentía y la sencillez con que empezó el cristianismo en el mundo. Sin licencia ni respaldo institucional, ni apoyo de poder humano alguno, sino tan solo de la motivación suscitada por la fuerza del Espíritu, recibido tras la resurrección de Jesucristo, expresados en su fundamental identidad comunitaria y misionera.

Este plano, que tiene por objeto recuperar la aleccionadora experiencia pública de la primera comunidad de seguidores de Jesús, tiene a su vez, como propósito, ayudar a percibir en tal gesto, la capacidad de los discípulos de haber hecho de tal proclamación solemne, el punto de partida con que irrumpió el cristianismo desde Galilea, y la fuente de sus motivaciones teológicas y de su autonomía. Hacer memoria de esta primordial y poco relevada experiencia kerygmática, es fundamental, para comprender el papel que jugó la teología en el origen y consolidación del cristianismo. 
El anuncio Kerygmático puede ayudar no solo a explicar la fuerza y la convicción de los comienzos, sino sobre todo, para apreciar la consistencia teológica con que los primeros fundamentaron su fe, despertando el interés y el entusiasmo por la aventura real comenzada por Jesús. El Kerygma, vale y moviliza, porque ayuda a comprobar que fue este espíritu de autonomía y de sentido crítico expresado con el anuncio del Kerygma, el que acompañó la necesidad de reflexionar teológicamente y así poder fortalecer y extender el sentido de pertenencia, continuada aquí y ahora, por su Iglesia.

El Kerygma, cuenta con una serie de dimensiones con que mostró la proclamación pública de que la muerte fue vencida por el crucificado 5 . Ella es el anuncio real y gozoso de que la desgracia y la maldad han quedado canceladas por Jesús, desde la cruz. Porque esta declaración pública ocurrió en el mismo lugar en que, poco antes de su muerte, Jesús 'desmercantilizó el Templo', a fin de que sus discípulos comprendieran posteriormente que testimoniarlo vivo y presente, significaba dedicarse a desacralizar el mercado y poner en sobreaviso sus persistentes formas de buscar convertir el ser humano en mercancía.

El Kerygma posee entre otras, las siguientes dimensiones que lo caracterizan: pneumatológica; histórica, primordial, pascual; crítica, profética, pública; comunitaria, solidaria, organizada; plural e intercultural; cristológica, laical y femenina.

\section{Plano Diocesano}

Tiene como fin, mostrar y difundir, el compromiso social y cristiano de los miembros de una diócesis, realizado en la época actual, reciente o históricamente ya lejana, dentro o fuera del país o de cualquier parte del mundo. Se encarga de dar a conocer el hecho de que la Iglesia Católica ha sido capaz de 'producir' hombres y mujeres que fueron capaces de poner la estructura de la Iglesia Católica, al servicio de la vida digna de los fieles, especialmente más indefensos de la sociedad. El plano diocesano se fundamenta en cinco aspectos fundamentales: el concepto de diócesis, el tipo de comunidad cristiana, los parámetros de funcionamiento pastoral, las funciones y la relación entre estructura y proceso. Paso a desarrollar brevemente cada una.

\subsection{Concepto de Diócesis}

En primer lugar, el concepto de diócesis, que fue redefinido a partir del Concilio Vaticano II y la reforma del Derecho Canónico, fue además identificado como Iglesia local o Iglesia particular, y puesto en vigencia a partir de 1983, de la manera siguiente:

"La diócesis es una porción del pueblo de Dios, cuyo cuidado pastoral se encomienda al Obispo con la cooperación del presbiterio, de manera que, unida a su pastor y congregada por él en el Espíritu Santo mediante el Evangelio y la Eucaristía, constituya una Iglesia particular, en la cual

5 Este tema fue adelantado en Dimensiones del Kerygma en una iglesia local, publicado en la revista Phainomenon de la UNIFE, Volumen 8, N 9 Enero-Diciembre 2009:140-173. 
verdaderamente está presente y actúa la Iglesia de Cristo una santa, católica y apostólica." (Derecho Canónico 1983: canon 369. El subrayado es mío) ${ }^{6}$.

De esta definición jurídica, puede desprenderse, en otras palabras, tres cosas: que la diócesis no es una 'parte' o fragmento pequeño o grande de Iglesia, sino una porción de toda la Iglesia. Y que cada diócesis es como el ADN de la iglesia total. Que, ella está formada por sus dos componentes básicos: los fieles y los pastores. Que los Pastores (obispo y clero, incluso religiosas y equipos de profesionales especializados), están puestos al servicio y cuidado del Pueblo sacerdotal de los fieles.

La plenitud del sacerdocio del obispo y el sacerdocio ministerial de los párrocos, sin embargo, confluyen en el Pueblo sacerdotal, que surge de "la aplicación del sacerdocio de Jesús (que es la vida misma y no una función determinada) a la comunidad en general (un pueblo sacerdotal) y a todos los cristianos en particular. No es un sacerdocio de segregación, sino de vinculación a los pecadores y de inmersión en el pueblo. No se trata de pretender privilegios sociales, sino de comprometerse con la entrega de Jesús."(Sotomayor-Fernandez, 2002:97).

Esta singular y muy poco tenida en cuenta categoría teológica de Pueblo Sacerdotal, fue por primera vez aplicada en el Perú, por el episcopado peruano, en el marco de su XXXVI Asamblea del 25 de enero de 1969, en la que el cardenal Juan Landázuri, como arzobispo cardenal y presidente de la Conferencia Episcopal del Perú, se propuso establecer las conclusiones pastorales de Medellín (1968), como la pauta paradigmática para renovar el funcionamiento pastoral de las diócesis del Perú. Al respecto, el antropólogo y teólogo Manuel Marzal precisó un detalle profundamente identitario del cristianismo:

"Muchas religiones aceptan entre sus miembros una fraternidad básica, que se funda en la imagen de la divinidad como padre y creador de todos; y otras, como la cristiana, hablan de «pueblo sacerdotal» que supera la dicotomía entre sacerdote y pueblo, donde éste debe acudir a aquél para acceder a la divinidad." (2002:37).

Este detalle aparentemente intrascendente, advertido por Marzal, es clave para la comprensión del sentido teológico profundo, no solo del quehacer teológico en sí, cuanto de la misma vida de la Iglesia localizada y animada por la identidad colectiva concretada en cada diócesis, entre otras, por las razones siguientes.

En primer lugar, porque para comprender en qué consiste el funcionamiento pastoral de una diócesis católica, hay que partir del axioma de la unidad y unicidad del sacerdocio cristiano, del que estructuralmente en su respectivo grado y esencia, están revestidos sus miembros, es decir, los pastores y los fieles. En segundo lugar, que el sacerdocio cristiano, dicotómico solo en apariencias de la jerarquía respecto del de los fieles, funda y explica sus raíces y su concepción unitaria en la

6 Como queda evidenciado, esta definición contenida en el Derecho Canónico, puesta en pie a partir de 1983, refiere y refleja explícitamente la constitución dogmática Lumen Gentium, dos décadas atrás aprobada y proclamada (24.11.1964). José M. Castillo, lo enfatiza: "El orden de capítulos de la Lumen Gentium es decisivo: ante todo, la Iglesia es presentada y comprendida como el nuevo Pueblo de Dios en su totalidad, pueblo sacerdotal, dentro del cual suscita el Espíritu diversidad de carismas y ministerios. Sólo después de esta presentación de conjunto, el Concilio habla de la jerarquía (LG 9-12, 18 ss.)”. 
práctica itinerante de un galileo laico y marginal como Jesús (Meier, 1997:37).

En tercer lugar, que el sacerdocio, en lugar de apartarlo y de separarlo del pueblo, -como ocurre con la mayoría de las religiones-, a Jesús le condujo a emerger desde abajo hacia la cruz, por haberle puesto en comunión, precisamente, con la vida y el destino de los impuros y marginales de su tiempo y de su sociedad; al punto de convertirlo en 'víctima' de ese mismo sacerdocio, pese a que su propósito haya sido "desmitificar la religión del templo. (Pagola, 2007: 454). instaurando y emprendiendo sobre el altar de la historia y de la vida cotidiana de la humanidad y del mundo, el proceso de "desacralización" y de conjura del "sagrado", "inexorable" y deshumanizante mercantilizado orden de cosas, "consagrado" a producir y reproducir cada vez más sofisticadamente solo mercancía humana.

El plano diocesano de la teología es el plano de la experiencia eclesial localizada, en el que los obispos y sacerdotes, resultan acreditando en su acción pastoral la calidad teológica del espíritu que les anima, de los principios y de los valores que mueven su cargo y para los que fueron consagrados, o ministerio, con que fundamentan, acompañan e impulsan, salvaguardan y custodian el Pueblo Sacerdotal.

\subsection{Comunidad cristiana}

En el plano diocesano, es de una importancia muy grande el tipo de comunidad cristiana caracterizada básicamente como solidaria, crítica y pública. Solidaria, porque sus miembros están dispuestos a solidarizarse con la necesidad o situaciones de sus hermanos más necesitados, ya sea de la propia o vecina comunidad, o incluso de otras diócesis y otros pueblos del mundo.
Crítica, porque, aunque se reconocen como miembros de una comunidad de base religiosa y confesional, en nombre de su identidad ciudadana, sus miembros participan de la marcha del barrio o de la administración de su municipalidad, en actividades como por ejemplo, el presupuesto participativo. Pública, porque siendo comunidades de tipo religioso, sus miembros no se conforman con vivir internamente en sus grupos o entre sus parroquias, sino que se interesan en desarrollar actividades de incidencia social y ciudadana.

\subsection{Parámetros de funcionamiento de una diócesis}

El funcionamiento pastoral e institucional de una diócesis está sujeto a tres parámetros principales: la imagen pública, de la comunión entre pastores y fieles, al servicio de los más pobres e indefensos de la diócesis.Es lo que en mi tesis doctoral, he podido reconocer, evidenciar y explicar que en la Arquidiócesis de Lima, el papel jugado por las motivaciones profundamente religiosas y particularmente católicas de los protagonistas de las sucesivas oleadas migratorias en el Cono Sur, fueron las mismas que definieron, tanto un nuevo tipo de comunidad cristiana que irrumpió y desarrolló, como unos nuevos parámetros de funcionamiento pastoral con que la Arquidiócesis de Lima respondió sin pausa de 1954 a 1996, a la renovación conciliar y al impacto local nacional de las Conferencias de Medellín, Puebla y Santo Domingo en torno a su área urbano marginal.

\subsection{Funciones de la Diócesis}

La función jurídica y organizativa de una diócesis, está cada una al servicio de la función pastoral. Y las tres, al servicio de la que he nombrado como función 
cósmica. La diócesis, no sólo desarrolla funciones administrativas, vale decir, organizativas y jurídicas. A partir de la invasión de Ciudad de Dios (Navidad de 1954), en mi tesis muestro que la Arquidiócesis de Lima, innovando, pudo desarrollar la función cósmica, mediante la cual, la comunidad de pastores y fieles consiguieron darle curso a su anhelo de trascendencia ejerciendo la impronta de comunión con el hombre y todos los hombres, 'desde abajo', y desde la vida privada cotidiana a la vida pública de los individuos y grupos de individuos de la comunidad cristiana.

Tal resultado se juega en la capacidad pastoral por la que, obispos y clero están obligados y para la que están ordenados en grado y esencia como miembros de la jerarquía de la Iglesia, para saber reconocer y legitimar con amplitud y respeto, para percibir y acoger con tino y creatividad de 'buen pastor', la impronta y la peculiar contribución del vasto sector poblacional de 'fieles', encomendado a su cuidado y auxilio.

¿En qué consiste la impronta y la peculiar contribución en general, del Pueblo Sacerdotal, y en particular, del sacerdocio común de los fieles, al funcionamiento de una diócesis? En constituir el componente mayoritario de la comunidad cristiana, en sostener con regularidad y continuidad el hacer realidad, desde abajo y desde la vida cotidiana, privada y pública, la impronta de comunión de la Iglesia. Los fieles, son portadores y fuente de proceso y de utopía pastoral, de la singularidad y de la identidad genuina y compleja que totaliza, otorga consistencia y solidez a una Iglesia particular o diócesis. Su variado estilo de presencia cotidiana y de experiencia protagónica en sus patrones de poblamiento, resulta 'localizando" la múltiple variedad de coordenadas y "demarcando" la heterogeneidad de límites, niveles, hitos y tramos simbólicos 'realmente reales' de sus múltiples escenarios, no sólo geográficos o históricos, jurídicos o únicamente administrativos de la diócesis que componen.

\subsection{Entre estructura y proceso}

En la marcha de una diócesis en renovación, después del Concilio Vaticano II, lo que la dinamiza no es el dilema entre estructura y proceso, en donde la estructura termina condicionando y paralizando la iniciativa y amordazando el proyecto de los actores, los fieles y los pastores. Esa dejó de ser la experiencia de la Arquidiócesis de Lima, a partir de 1954 a 1996. Porque, a partir de la renovación conciliar y la de Medellín, la Iglesia de Lima empezó a comprender que la estructura es más simbólica que real, y que no existe de manera independiente. Por lo que, en nombre del Pueblo sacerdotal conformado por los fieles presidido por los pastores, puso la estructura al servicio del proceso y la utopía pastoral, del proyecto de los protagonistas de la diócesis: los fieles y los pastores.

En la Arquidiócesis de Lima, a partir de 1954 a 1996, por primera vez, en toda la historia de la Iglesia en el Perú, fuera, o por lo menos lejos, de un catolicismo autoritario, colonial y de conquista, irrumpió incipiente, no otra iglesia, sino la estructura diocesana y el proceso pastoral de otro catolicismo. Aunque esta vez, un catolicismo cuyos pastores y fieles, fueron capaces de poner la estructura y la tradición de la diócesis, al servicio de la utopía pastoral y de la impronta de comunión que vinieron experimentando como Iglesia. Un catolicismo, que impulsado por la exclusiva iniciativa de los pobladores, 
constituyó y definió por un lado, una identidad adulta y fraterna de Pueblo sacerdotal, regio y profético; a la vez por otro, mostró consumada, y desde abajo, la naturaleza integratoria de la comunidad -pastores, junto a fieles-, de la que fue infundida la arquidiócesis de Lima.

En otros términos, lo que el funcionamiento pastoral de una diócesis puede mostrar es que entre la estructura o tradición y el proceso o los actores y su utopía pastoral, puede haber más que un dilema un complemento, una integración. Un complemento en el que la estructura y su función legitimadora, quedan puestas al servicio de los anhelos pastorales, y sus procesos, pese a las crisis.

\section{Conclusión}

El modelo de funcionamiento pastoral desarrollado por la Arquidiócesis de Lima, y que propició la concepción y el nacimiento de la Diócesis de Lurín, puede servir para evidenciar el tipo de reflexión teológica que acompañó ese proceso. Y da pie para comprender y apreciar el lugar y el papel que representan para la reflexión teológica, casos aparentemente ajenos y aislados como los de muchas diócesis de América Latina, cuya historia desconocemos. Por ejemplo, el que Román Lejtman recoge de sus informantes respecto del episcopado católico argentino.

En particular, del testimonio de Enrique Angelelli, un obispo que, ordenado auxiliar por Juan XXIII en 1960, y enviado a La Rioja por Pablo VI en 1968, hizo de su diócesis una oportunidad para la renovación conciliar y la transformación pastoral post-Medellín. Un obispo que, mientras logró permanecer con vida, hizo de su diócesis un espacio de humanidad y de resistencia pública. Y que incluso, una vez amenazado y hasta silenciado para siempre, se convirtió en símbolo y mártir de una Iglesia que sin esperar la aprobación del Estado ni el respaldo de sus colegas obispos, continúa jugándose el prestigio y la misma vida para demostrar que desde su mismo rebaño riojano, para todo su país y América Latina, los argentinos indefensos pudieron y pueden contar con el pastor que este abandonado pueblo requería a gritos, al punto de conseguir desarrollar y establecer desde su iglesia local la opción preferencial por los más pobres, los indefensos, los desaparecidos. Lejtman anuncia en su reportaje que ha sido abierto el proceso de canonización del obispo ${ }^{7}$.

En un caso actual y muy nuestro, es de agradecer el fresco y revitalizante testimonio pascual del arzobispo de Huancayo, Pedro Barreto, Arzobispo de la Arquidiócesis de Huancayo y Vicepresidente de la Conferencia Episcopal Peruana, Presidente del Departamento de Justicia y Solidaridad del Consejo Episcopal Latinoamericano CELAM. Porque, el testimonio de este obispo y de su diócesis entera, no solo a mis alumnas, sino a la comunidad católica y cristiana, y a la sociedad entera de Perú y el Continente, nos está ayudando a darle vida tanto a las fiestas litúrgicas, como sobre todo a la conciencia y a la misión no negociable que la Iglesia renueva cada día con el mismo Señor. Nos está enseñando, a comprender que lo único que por ejemplo, hace 'santa' a una semana, es el seguimiento valiente de Jesús. Que la honesta y sincera manera de vivir y desearse la Pascua del Vencedor de la Muerte, es celebrarla, dejando de lado otros asuntos ajenos

7 Documenta El martirio de Angelelli, Video de Roman Lejtman, 2011. 
al papel del pastor, consolidando el 'cuidado pastoral' de los más indefensos, evidenciando de manera pública la solidaridad con los insignificantes de su Iglesia particular, aunque exponiendo la propia integridad; compartiendo el destino y sobre todo la esperanza y el derecho a existir dignamente, de tantos peruanos ciudadanos que aún, no cuentan para las grandes y solemnes decisiones de quienes se sienten mejor ubicados en esta actual sociedad.

\section{Efectivamente, la experiencia} diocesana, puede ser útil para iniciar a nuestras alumnas en la reflexión teológica, porque ella permite propiciar hasta una relación más profunda e interpersonal con quien ha sido amenazado de muerte, por defender la vida y la salud de los fieles de su diócesis. Tal vez por eso, tal amenaza no ha sido ninguna noticia "seria" para los medios oficiales y sus 'sagradas leyes' del mercado. Pero lo es suficientemente digna para quienes intentamos emprender el análisis y la comprensión de la reflexión teológica, como hasta para poder decirle cordialmente:

"Desde nuestras aulas, apreciamos, hermano Pedro, tus profundas y sólidas motivaciones teológicas, al verte muy lejos y distante de haber caído en la tentación de la complicidad con los poderes actuales, que como siempre, creen poderlo comprar todo con el oro mal habido, incluso la conciencia y la voz del pastor libre y apasionado por la vida de su rebaño. iGracias a ti, hermano Pedro!, iPastor bueno del Reino de Dios y de su justicia! Somos testigos privilegiados de tu compromiso asumido, de seguir los pasos de Monseñor José Dammert, obispo emérito de Cajamarca, permanentemente distinguido por su valiente voz pública. Aunque te persigan y amenacen, con tu heroico y arriesgado testimonio, real y simbólicamente ya conseguiste dar por cancelada la muerte en todos y cada uno de los centros mineros del país y del Continente, donde solo importa el crecimiento y para nada la dignidad afectada de los pobladores humildes de los alrededores."

No obstante, también es muy cierto que, en el plano diocesano encontramos, no solo el testimonio del obispo valiente, o solo de los sacerdotes sacrificados y las religiosas generosas, sino, además y sobre todo, la vida de una población en general, particularmente considerada como la de "los más alejados". Y cuya situación en el caso de la aún joven Diócesis de Lurín, han resultado convirtiéndose en motivo y fuente de conversión pastoral, al punto de haberles ayudado a descubrir a los agentes pastorales de que pastoralmente hablando, los alejados fueron más bien los párrocos y catequistas de las parroquias, los que abandonando las "99 ovejas", inconscientemente prefirieron quedarse con las pocas que no dejan de frecuentar el templo.

Al parecer, la reflexión teológica en nuestras aulas, resulta cobrando vida e ingenio, cada vez que se la asocia y articula a la vida, pasión, muerte y resurrección de la mayor parte de las diócesis, en donde el énfasis en la solidaridad ayudó a fortalecer los lazos de cooperación dentro de las comunidades afectadas por problemas como el terrorismo, en los Andes, en la selva y en los barrios populares de las ciudades. En la doble década del terror, el acento puesto en la defensa de la vida fue una orientación clara. ¿Cuál fue la teología que inspiró y motivó a muchos obispos, sacerdotes, religiosas, catequistas y laicos comprometidos que acompañaron a la población frente a la amenazas de los terroristas y de las fuerzas del orden? Este modo de comprometerse surge 
del Concilio Vaticano II (62-65) y su aplicación en las diferentes regiones." ${ }^{8}$

En definitiva, el plano diocesano, cargado de abundante experiencia pastoral continental, particularmente profética y martirial, ocurrida a lo largo de la segunda mitad del siglo XX, puede contribuir a comprender no solamente los temas teológicos, ni solo el caminar mismo de la Iglesia y su Doctrina Social, sino sobre todo a despertar la adhesión y el seguimiento de la persona misma de Jesús, el Señor del Reino de Vida, Justicia y Paz. De esa experiencia vivida en el país por nuestras diócesis, durante la doble década de terror y violencia, es de la que hoy podemos sacar mucho. Además de lecciones, la capacidad teológica de movilizar la vitalidad, convocar a la creatividad y a verter la inspiración para poder actuar localizada y contextualmente. Con el plano bíblico y el plano kerygmático de la vida y de la fe que brinda la reflexión teológica, y que la CVR empezó a analizar e interpretar en su Informe Final en los significativos términos siguientes:

"La Comisión de la Verdad y Reconciliación (CVR) ha encontrado que, durante el período de la violencia en el Perú, la Iglesia Católica desempeñó un importante papel de acompañamiento y protección de los peruanos golpeados por la violencia de las organizaciones subversivas y de las fuerzas de seguridad del Estado. Fue en numerosas regiones del país una voz de denuncia de los crímenes y las violaciones de los derechos humanos y proclamó y defendió el valor de la vida y la dignidad de la persona. La mayoría de los obispos, sacerdotes y religiosas, así como multitud de laicos y laicas, constituyeron una fuerza moral y una fuente de esperanza (...).

\section{REFERENCIAS:}

BENTUÉ, A. (1996) Grandes teólogos del siglo XX. Chile: San Pablo.

BERZOSA, R. (1999) ¿Qué es teología? Una aproximación a su identidad y a su método. Madrid: Desclee de Brouwer.

MEIER, J. (1997) Un judío marginal. Nueva visión del Jesús histórico. Navarra: Verbo. Divino.

LIBANIO, J. B. (2009) Introducción a la Teología. Perfil, enfoques, tareas. México: Dabar.

MARZAL, M. (2002) Tierra Encantada. Tratado de antropología religiosa de América Latina. Lima: PUCP.

PAGOLA, J. A. (2007) Jesús. Aproximación histórica. Madrid: PPC.

SOTOMAYOR, M. y FERNÁNDEZ, J. (Eds) (2002) Historia del Cristianismo I. El mundo antiguo. Madrid: Trotta

VILANOVA, E. (2000) Para comprender la Teología Navarra: Verbo Divino. 